$10-2015$

\title{
Multitrophic functional diversity predicts ecosystem functioning in experimental assemblages of estuarine consumers
}

JS Lefcheck

Virginia Institute of Marine Science

JE Duffy

Virginia Institute of Marine Science

Follow this and additional works at: https://scholarworks.wm.edu/vimsarticles

Part of the Ecology and Evolutionary Biology Commons, and the Marine Biology Commons

\section{Recommended Citation}

Lefcheck, JS and Duffy, JE, "Multitrophic functional diversity predicts ecosystem functioning in experimental assemblages of estuarine consumers" (2015). VIMS Articles. 1734.

https://scholarworks.wm.edu/vimsarticles/1734 


\title{
Multitrophic functional diversity predicts ecosystem functioning in experimental assemblages of estuarine consumers
}

\author{
Jonathan S. LeFCheCK ${ }^{1,3}$ And J. Emmett DuFfy ${ }^{1,2}$ \\ ${ }^{1}$ Department of Biological Sciences, Virginia Institute of Marine Science, The College of William and Mary, Gloucester Point, \\ Virginia 23062-1346 USA \\ ${ }^{2}$ Tennenbaum Marine Observatories Network, Smithsonian Institution, Washington, D.C. 20013-7012 USA
}

\begin{abstract}
The use of functional traits to explain how biodiversity affects ecosystem functioning has attracted intense interest, yet few studies have a priori altered functional diversity, especially in multitrophic communities. Here, we manipulated multivariate functional diversity of estuarine grazers and predators within multiple levels of species richness to test how species richness and functional diversity predicted ecosystem functioning in a multitrophic food web. Community functional diversity was a better predictor than species richness for the majority of ecosystem properties, based on generalized linear mixedeffects models. Combining inferences from eight traits into a single multivariate index increased prediction accuracy of these models relative to any individual trait. Structural equation modeling revealed that functional diversity of both grazers and predators was important in driving final biomass within trophic levels, with stronger effects observed for predators. We also show that different species drove different ecosystem responses, with evidence for both sampling effects and complementarity. Our study extends experimental investigations of functional trait diversity to a multilevel food web, and demonstrates that functional diversity can be more accurate and effective than species richness in predicting community biomass in a food web context.
\end{abstract}

Key words: biodiversity; Chesapeake Bay; consumers; ecosystem functioning; estuaries; functional diversity; grazers; predators.

\section{INTRODUCTION}

Hundreds of experiments have shown that biodiversity generally enhances the functioning of ecosystems, including biomass production, efficiency of resource use, and nutrient cycling, yet there are many examples where diversity has had a neutral or even negative effect on functioning (Hooper et al. 2005, Cardinale et al. 2006, 2012, Lefcheck et al. 2013, Gamfeldt et al. 2015). A possible explanation for the prevalence of negative diversity effects is that the species used in these manipulations overlap sufficiently in their ecological strategies to prevent mechanisms like resource use partitioning from occurring (Hooper et al. 2005). One way to characterize the degree of redundancy among species is to consider their functional traits, aspects of their morphology, physiology, phenology, and behavior that distinguish ecological differences among species. The variation in these traits across all species within an assemblage can be used to characterize functional trait diversity (hereafter FD).

There has been a great deal of interest in using FD to predict ecosystem functioning because traits not only

Manuscript received 16 October 2014; revised 24 March 2015; accepted 13 May 2015. Corresponding Editor: J. H. Grabowski.

${ }^{3}$ E-mail: jslefche@vims.edu account for potential functional redundancy (Rosenfeld 2002), but also provide a mechanistic link to observed diversity effects (Díaz and Cabido 2001). Recent investigations have integrated multiple traits into multivariate indices of $\mathrm{FD}$, which have yielded varying support for the utility of FD as a predictor of ecosystem functioning, principally standing stock biomass (Petchey et al. 2004, Flynn et al. 2011, Mouillot et al. 2011, Gagic et al. 2015). However, most experimental studies utilizing multivariate FD have taken a post hoc approach by applying trait data to existing richness manipulations, predominantly of grassland plants. This approach can lead to ambiguous results if the replicates within and across levels of richness were not sufficiently varied in terms of their functional traits. Only a few studies have a priori manipulated multiple traits (e.g., Schittko et al. 2014), and two used at most pairwise combinations of aquatic algae species (Griffin et al. 2009, Shurin et al. 2014), which is not generally recognized as a diversity manipulation per se (Cardinale et al. 2006).

Furthermore, much of biodiversity-ecosystem-function research has been conducted with terrestrial plants, and an important challenge is understanding the consequences of changing diversity in complex natural food webs (Duffy et al. 2007, Reiss et al. 2009). Comparatively few studies have simultaneously manipulated the species richness of adjacent trophic levels 
(e.g., both predators and prey), and those that have done so generally found a strong role of consumer diversity for the structure and functioning of lower trophic levels (Fox 2004, Gamfeldt et al. 2005, Bruno et al. 2008, Douglass et al. 2008). This strong top-down effect of consumer diversity has often been shown to depend on feeding biology, specifically whether the consumers are omnivorous (Bruno and O'Connor 2005) or intra-guild predators (Finke and Denno 2004), or whether they vary in their per capita consumption rates (Straub and Snyder 2006) or resource preferences (O'Connor and Bruno 2007). While these studies suggested differences in feeding ecology among species as a potential explanation for their results, they did not directly manipulate resource acquisition strategies, but rather generally assumed that feeding diversity would be correlated with species richness. Of the three prior studies that a priori manipulated consumer traits within a single level of richness, two found variation in trophic ecology to be a strong predictor of resource depletion (Schmitz 2008, Best et al. 2013), while one found no effect (O’Connor and Bruno 2009).

In this study, we manipulated multivariate community FD of consumers based on eight functional traits both within and across multiple levels of species richness in experimental estuarine mesocosms. The consumers included naturally abundant herbivorous grazers and their predators, which allowed us to experimentally recreate a model estuarine food web. We expected multivariate FD to be a better predictor of ecosystem properties than species richness by capturing a wider range of variation in ecological strategies (Petchey and Gaston 2002). Further, we expected FD within a trophic level to enhance the biomass of that trophic level (Duffy et al. 2007), and for predator diversity to have a stronger top-down effect than the bottom-up effect of grazer diversity (Gamfeldt et al. 2005, Borer et al. 2006, O'Connor and Bruno 2007, Douglass et al. 2008).

\section{Methods \\ Experimental species}

We defined a nine-species pool based on natural abundances of herbivores and their predators sampled over 15 years in the York River Estuary, Chesapeake Bay, Virginia, USA (Douglass et al. 2010, Lefcheck 2015). The herbivores included three crustacean mesograzers: the amphipods Gammarus mucronatus and Cymadusa compta (potentially including a lesser incidental number of ampithoid amphipods, so referred to here as Ampithoid spp.), and the isopod Erichsonella attenuata. All three species are key grazers in the Chesapeake Bay and represent important trophic links in the local food web (van Montfrans et al. 1984). We also used one gastropod, Bittiolum varium, a relatively small but seasonally abundant mesograzer (Duffy et al. 2003). The final herbivore was the shrimp Hippolyte pleuracanthus, whose diet is mainly micro- and macroalgae, but which occasionally includes animal tissue
(Douglass et al. 2011). The predators included the grass shrimp Palaemonetes pugio and juvenile blue crab Callinectes sapidus (30-50 mm carapace width), both of which are omnivorous (Douglass et al. 2011), as well as the pipefish Syngnathus spp. and mummichog Fundulus heteroclitus. Trophic guilds were assigned using existing stable isotope data (Douglass et al. 2011). For all of these species, we scored eight functional traits relating to morphology (defense, mobility, mean and maximum biomass, body plan), feeding habits (trophic level), and life history and phenology (reproductive mode, month of maximum abundance in the estuary), with both direct and indirect consequences for ecosystem functioning (Appendix A: Table A1). All traits used in this study have been proposed to have a strong link to ecosystem function (Bremner et al. 2003).

\section{Experimental design}

We employed a semi-nested design manipulating high and low multivariate FD within three- and six-species assemblages, as well as each species by itself (one species) and the multispecies polyculture (nine species; Fig. A1). To characterize FD, we chose the index of functional richness (Villéger et al. 2008). Functional richness quantifies the absolute volume of trait space occupied by all species within an assemblage. It is the volume of an $n$-dimensional polygon whose vertices are defined by the most functionally extreme species (Fig. A2). We chose functional richness as our index of FD because it does not take into account relative abundances. This behavior is ideal for our experiment, which combines large but rare predators with small but abundant grazers. Hereafter, when we refer to functional diversity (FD), we mean functional richness. Functional richness was calculated using minor modifications to the dbFD function in the FD package (Laliberte et al. 2014; see Supplement).

Within the two intermediate diversity levels, we generated every possible combination of three and six species. We calculated FD for each of these 168 combinations, and then randomly drew six replicates from the lower 25th percentile to represent low FD, and six replicates from the upper 75th percentile to represent high FD for three- and six-species treatments, respectively. We discarded and redrew any three-species replicates that contained all predators, as we wanted to ensure resource availability for all multispecies replicates. Six additional replicates for each of the nine single-species treatments and the nine-species mixture yielded a total of $N=84$ replicates. In each treatment, we equalized the initial biomass of the grazers at densities comparable to those observed in the field, and those used in previous mesocosm experiments with these organisms (Duffy et al. 2003, 2005). As a consequence of their large size and the logistical constraints on equalizing biomass, each predator was simply stocked with a single individual in the treatments 
in which it appeared, and its initial mass recorded to include as a covariate in subsequent statistical analyses.

In May 2012, experimental assemblages were created in 19-L mesocosm buckets placed in six flow-through seawater tanks. Water was passed through $150-\mu \mathrm{m}$ mesh filters, which minimized the introduction of non-target species while permitting the passage of smaller invertebrate larvae (recruits) such as barnacles (Balanus spp.), bubble snails (Haminoea solitaria), polychaetes (Nereis spp.), and tunicates (Mogula manhattensis), as well as propagules of green and red filamentous algae. Mesocosms were arranged in a block design, with one replicate of each of the 14 treatments present in a single tank. Each mesocosm was filled with $1 \mathrm{~kg}$ of crushed oyster shell to provide a natural substrate, and $30 \mathrm{~g}$ wet mass of the macroalgae Gracilaria spp. (hereafter Gracilaria). Gracilaria is a common drift macroalgae in the Chesapeake Bay, and harbors a diverse epifaunal community (Parker et al. 2001). Gracilaria were defaunated in a diluted solution of the commercially available pesticide Sevin (Gardentech, Atlanta, Georgia, USA) before being placed into the mesocosms for $72 \mathrm{~h}$ prior to introduction of any animals, after which time, grazers were introduced, followed $48 \mathrm{~h}$ later by the predators. Twice a week, a pinch of freeze-dried krill was introduced into every mesocosm to prevent starvation of predators in monoculture.

The experiment was terminated after three weeks when we observed near total consumption of Gracilaria in some replicates. All algal and animal material was removed from the mesocosms and frozen, and predator wet masses were measured. Later, Gracilaria, recruiting red and green filamentous algae, predators, and recruiting invertebrates were thawed and identified to species, dried at $60^{\circ} \mathrm{C}$ until mass was stable, and then combusted to obtain final ash-free dry mass (AFDM) of each taxon. Smaller invertebrates, such as the stocked grazers and polychaetes, were isolated and passed through a series of stacked sieves, sorted to species, and counted. Abundance of each taxon in each sieve size was converted to an estimate of AFDM using the equations in Edgar (1990). Two replicates (one each of $C$. sapidus and $F$. heteroclitus monocultures) were discarded due to contamination, and one replicate was lost during the experiment breakdown (nine-species polyculture), leaving a total of $N=81$ replicates for analysis. Vertebrates were handled according to IACUC standards [protocol 2012-05-11-7960, administered through the College of William and Mary].

\section{Statistical analysis}

To quantify the relative contributions of initial species richness vs. FD in explaining ecosystem responses, we constructed generalized linear mixed-effects models (GLMMs) regressing each response against species richness or FD, allowing the intercept to vary by tank. For final predator biomass, an additional covariate of initial predator biomass was included in the model, since predator biomass could not be equalized at the start of the experiment. Species richness and FD were evaluated singly to avoid issues with multicollinearity. We selected the best model using AIC (Burnham and Anderson 2002). We also calculated marginal and conditional $R^{2}$ values (sensu Nakagawa and Schielzeth 2012), corresponding to the variance explained by the fixed effect and the combined fixed and random effects, respectively, to gain a sense of the approximate variance in the response explained by each of the two predictors. We additionally fit regressions of each response against richness, FD, and their interaction, knowing that resulting $P$ values are likely to be inflated due to observed collinearity between richness and FD. All models were constructed in the $\mathrm{R}$ package nlme (Pinheiro et al. 2013). Model assumptions, including homogeneity of variance and normality of errors, were assessed graphically. Diversity indices were scaled by mean and variance to better meet model assumptions. Even so, for several responses, residuals were highly heteroscedastic. For these responses, we modeled the variance using the function varIdent, using initial species richness levels as the stratum. Marginal and conditional $R^{2}$ values were calculated using the function by Lefcheck and Casallas (available online). ${ }^{4}$

As multivariate FD may obscure the potentially interacting contributions of individual traits (e.g., Spasojevic and Suding 2012), we conducted two additional analyses to assess the role of individual traits in explaining the observed patterns. First, we calculated the functional richness index separately for each individual trait, essentially representing the range of values encompassed by a particular assemblage for that trait. We then regressed these univariate $\mathrm{FD}$ values against each ecosystem response. This procedure allowed us to quantify the contributions of individual traits and determine whether trade-offs existed in the magnitude and direction of their individual effects. Second, we assessed the contribution of individual traits to the multivariate effect by conducting a jack-knifing procedure that removed a single trait, recalculated a multivariate FD from the remaining seven traits, and regressed this reduced jack-knifed index against each ecosystem response. We then refit the GLMMs to these jack-knifed indices and compared them to the GLMMs regressing the full multivariate index using Akaike's information criterion (AIC). The change in AIC score between the jack-knifed vs. the full index of FD ( $\triangle \mathrm{AIC})$ indicated whether any trait(s) had an inordinate influence on multivariate FD.

Because there was a potentially complex network of interactions among variables in the experiment, we conducted piecewise structural equation modeling (SEM). Piecewise SEM combines information from multiple separate linear models into a single causal

${ }^{4}$ https://github.com/jslefche/rsquared.glmer 
TABLE 1. Akaike's information criterion (AIC) scores, marginal $R^{2}\left(R_{\mathrm{m}}^{2}\right)$, and conditional $R^{2}\left(R_{\mathrm{c}}^{2}\right)$ values for competing models containing either species richness or functional diversity (functional richness) as a predictor of five ecosystem responses across three trophic levels.

\begin{tabular}{|c|c|c|c|c|c|c|}
\hline \multirow[b]{2}{*}{ Response } & \multicolumn{3}{|c|}{ Species richness } & \multicolumn{3}{|c|}{ Functional diversity } \\
\hline & $\mathrm{AIC}$ & $R_{\mathrm{m}}^{2}$ & $R_{\mathrm{c}}^{2}$ & $\mathrm{AIC}$ & $R_{\mathrm{m}}^{2}$ & $R_{\mathrm{c}}^{2}$ \\
\hline Final grazer biomass & 133.1 & 0.106 & 0.107 & 129.3 & 0.167 & 0.168 \\
\hline Final predator biomass & 31.4 & 0.479 & 0.479 & 25.4 & 0.534 & 0.534 \\
\hline Recruiting invertebrate biomass & -52.9 & 0.152 & 0.173 & -55.3 & 0.233 & 0.274 \\
\hline Final algal biomass & -222.6 & 0.003 & 0.022 & -222.3 & 0.000 & 0.018 \\
\hline Final Gracilaria biomass & 288.5 & 0.063 & 0.063 & 288.9 & 0.059 & 0.059 \\
\hline
\end{tabular}

Notes: Models that were significantly better than the other at explaining the response based on lower AIC scores are shown in boldface type. Models predicting algal or Gracilaria biomass were nearly equivalent, and thus those rows have no cells with boldface text.

network (Shipley 2009). Because the individual models can incorporate random structures and non-normal distributions, piecewise SEM is a powerful and flexible alternative to traditional variance-covariance-based SEM. SEM allowed us to decompose the relative contributions of herbivore vs. predator diversity on ecosystem responses to test whether predators were wholly responsible for the significant community FD effects observed in our GLMMs. Following the recommendations of Grace (2006) and Grace et al. (2012), we constructed a single causal network using knowledge of the system and ecological theory to define the paths of interest (described in further detail in Appendix B). We fit the component models as GLMMs. We ran this model twice, substituting either species or functional richness for variables relating to herbivore or predator diversity. Overall fit was assessed using Shipley's test of $d$-separation, which yields a Fisher's $C$ statistic that is $\chi^{2}$ distributed (Shipley 2009). Species vs. functional richness SEMs were compared using AIC (Shipley 2013). Coefficients reported in the text are scaled by means and standard deviations so that comparisons can be made across responses of varying units. For these and all other analyses, we held an experiment-wide $\alpha=0.05$. We used the open-source $\mathrm{R}$ package piecewiseSEM to conduct the piecewise SEM (version 0.9 ; available online) ${ }^{5}$

We further modeled the contribution of each individual species to understand whether species with different combinations of traits influenced different ecosystem functions. We constructed GLMMs regressing each response against the presence/absence of each species (e.g., Isbell et al. 2011). To understand whether the strongest effects were the result of extreme combinations of traits, we regressed the effect sizes from the GLMMs against functional distinctness, calculated as the average pairwise functional distance between a given species and all other species. Distances were derived from Gower's metric (Podani 1999), which unites both continuous and categorical trait

${ }^{5}$ https://github.com/jslefche/piecewiseSEM information into a single continuous measure. All data and $\mathrm{R}$ code are provided as supplements.

\section{Results}

Multivariate functional diversity (FD) was a better predictor of and explained more variance in predator, grazer, and recruiting invertebrate biomass than species richness, based on comparison of model AIC values and marginal and conditional $R^{2}$ values (Table 1). Neither diversity index significantly predicted functions related to primary producers, explaining only $3-6 \%$ of the variance in recruiting algal and Gracilaria biomass. Despite the collinearity between initial species richness and FD (Appenidx A: Fig. A3) leading to conservative $P$ values, models regressing the same responses in Table 1 against species richness, FD, and their interaction as predictors revealed identical trends to the model selection presented above (Table A2). Predicted fits extracted from the interaction models revealed a weaker but significant decline in final grazer biomass with increasing FD (Fig. 1a), presumably due to the increasing frequency of predators as FD increased. Recruiting invertebrate biomass also declined with increasing FD (Fig. 1b), also presumably indicating direct consumption by predators and omnivorous grazers (e.g., Duffy et al. 2003). Final predator biomass was higher in mesocosms with higher FD, even after accounting for differences in initial predator biomass (Fig. 1c). As found during the model selection procedure, there was no relationship between FD and either recruiting algal biomass (Fig. 1d) or final Gracilaria biomass (Fig. 1e).

Exploration of the effects of individual traits on final biomass responses revealed similar trends to multivariate FD (Fig. 2). These general trends were also conserved in our jack-knifing exercise, in which traits were individually removed and multivariate FD was calculated from the remaining pool of traits. The one exception was final predator biomass, which was more poorly predicted when either body plan, trophic level, or reproductive mode were left out, and better predicted when mobility and phenology (month of maximum abundance) were omitted (Table A3). Interestingly, the confidence intervals derived from multivariate FD were 

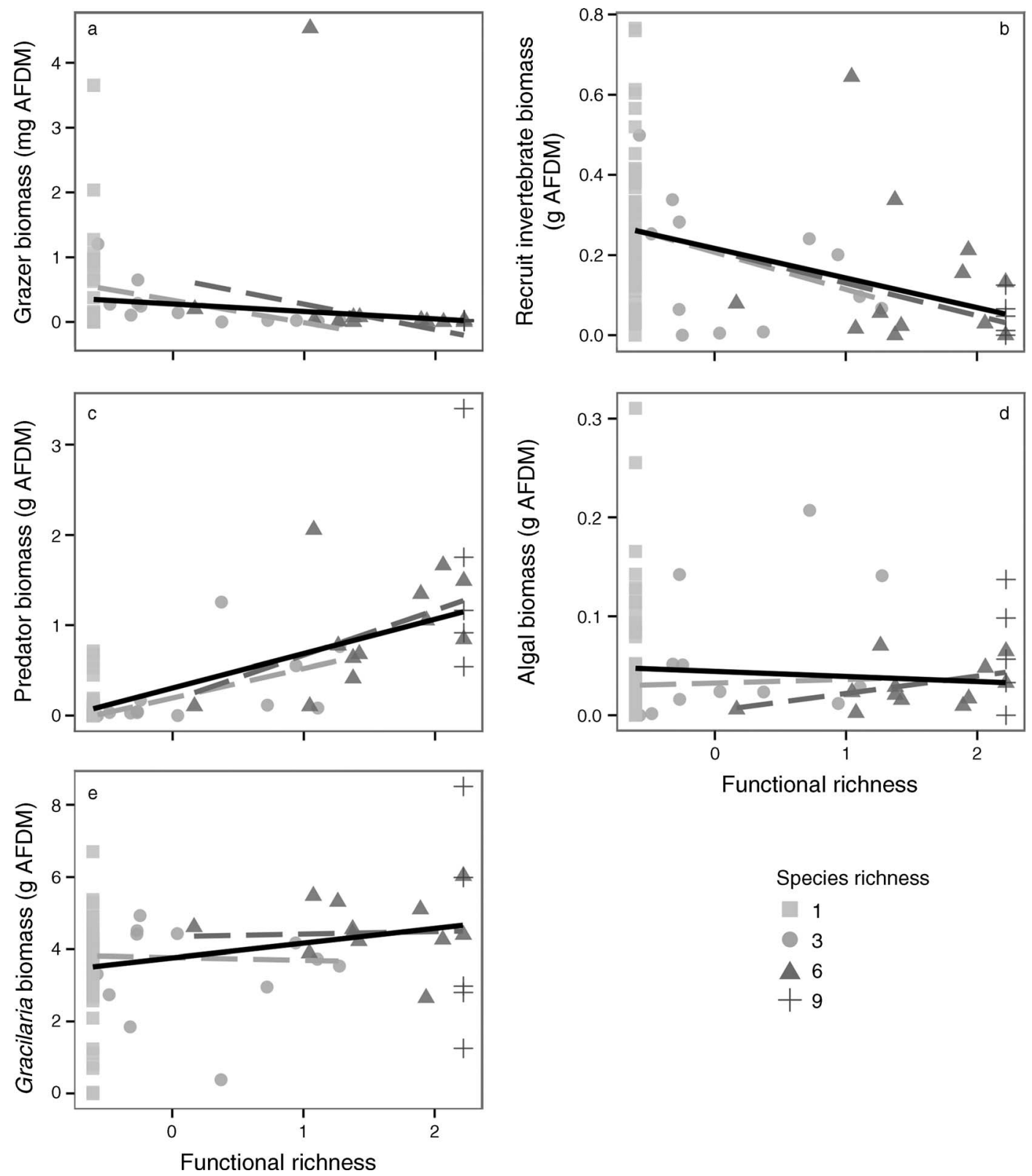

Functional richness

Species richness

$\square 1$

3

$\Delta 6$

$+9$

FIG. 1. Scatterplots of initial functional richness against each ecosystem response. Symbols correspond to the richness level (one, three, six, or nine species). Gray lines represent predicted fits from a general linear mixed effects model for three- (light gray) and six-species (dark gray) treatment. The black line represents the overall trend across all richness levels from the same model. The regression of final predator biomass against functional richness (panel c) included initial predator biomass as an additive covariate. AFDM, ash-free dry mass.

narrower than for individual traits, particularly for armor, trophic level, mobility, and reproductive mode, suggesting that the composite index across multiple traits improved accuracy in predicting community biomass (Fig. 2, Fig. A4). Together, these results suggest that the inferences derived from multivariate FD were generally more robust than those for individual traits, and qualitatively corresponded with those derived from univariate FD.
To determine whether the positive effects of FD from the model fitting procedure persisted when partitioned by trophic level, we fit a structural equation model (SEM) decomposing community FD into independent herbivore and predator FD effects. We also fit the same model replacing FD with species richness. Overall, the FD SEM fit the data extremely well $\left(C_{36}=24.65, P=\right.$ 0.924 , Fig. 3), and revealed that the strongest relationship occurred between initial predator FD and final 


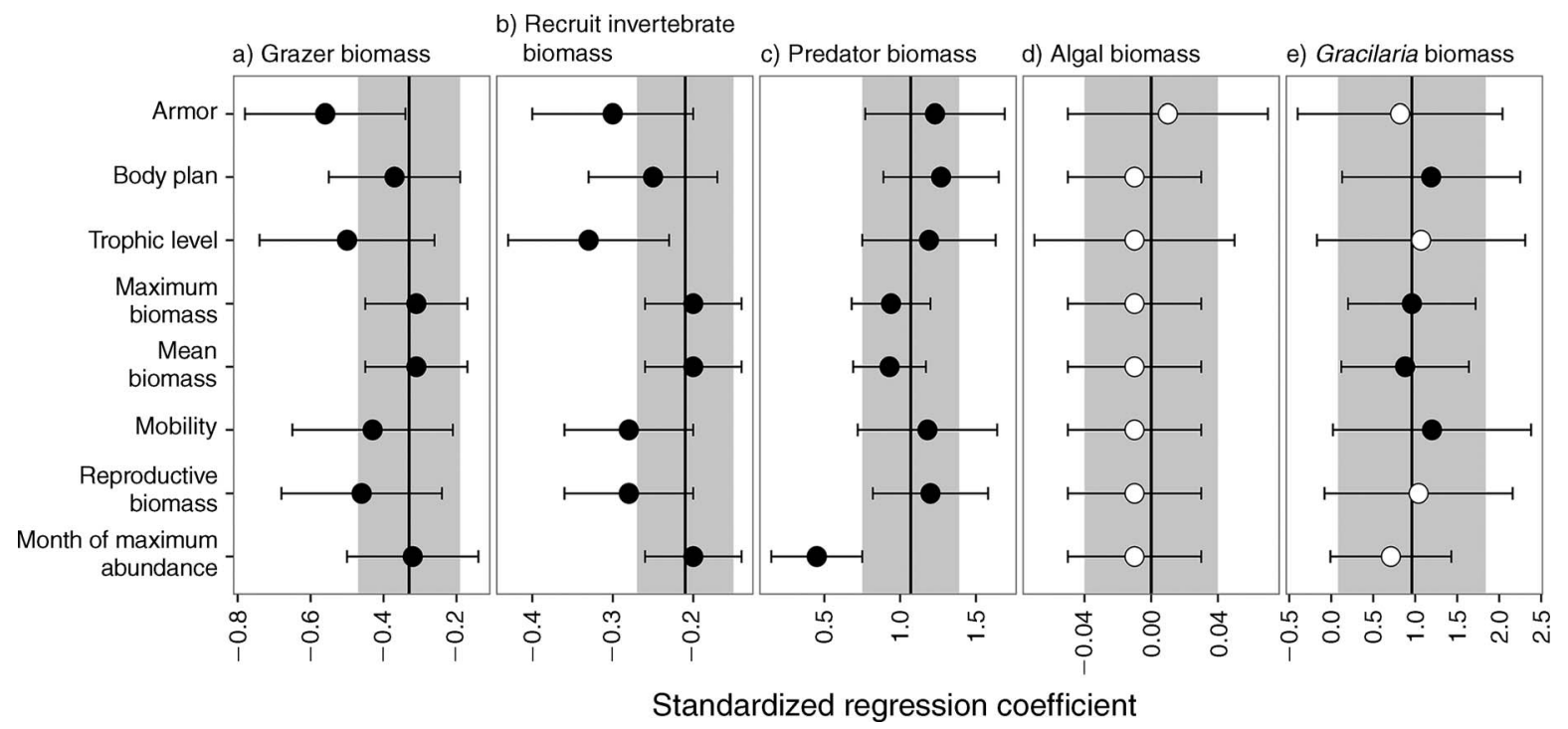

FIG. 2. Standardized regression coefficients from models regressing each ecosystem response (panel labels) against functional richness calculated using each functional trait (listed on $y$-axis) individually. Points are linear estimates $\pm 95 \%$ confidence intervals (2SE). Solid points indicate significance $(P<0.05)$, while unfilled points are nonsignificant $(P \geq 0.05)$. Vertical lines represent the linear estimates for models regressing the multivariate index of functional richness (including all traits), and shaded areas indicate $\pm 95 \%$ confidence intervals.

predator biomass (standardized $\beta=0.464, P<0.001$, Fig. 3), after controlling for initial predator biomass. This relationship was still significant and similar in magnitude when considering only replicates that contained predators $(\beta=0.419, P=0.012)$, to omit the influence of many replicates with 0 values for predator diversity. We also observed a positive but weaker relationship between final grazer FD and final grazer biomass ( $\beta=0.142, P=0.006$, Fig. 3 ), even after the predator effects on grazer biomass were taken into account. This trend can be better visualized by extracting the partial correlations between final grazer

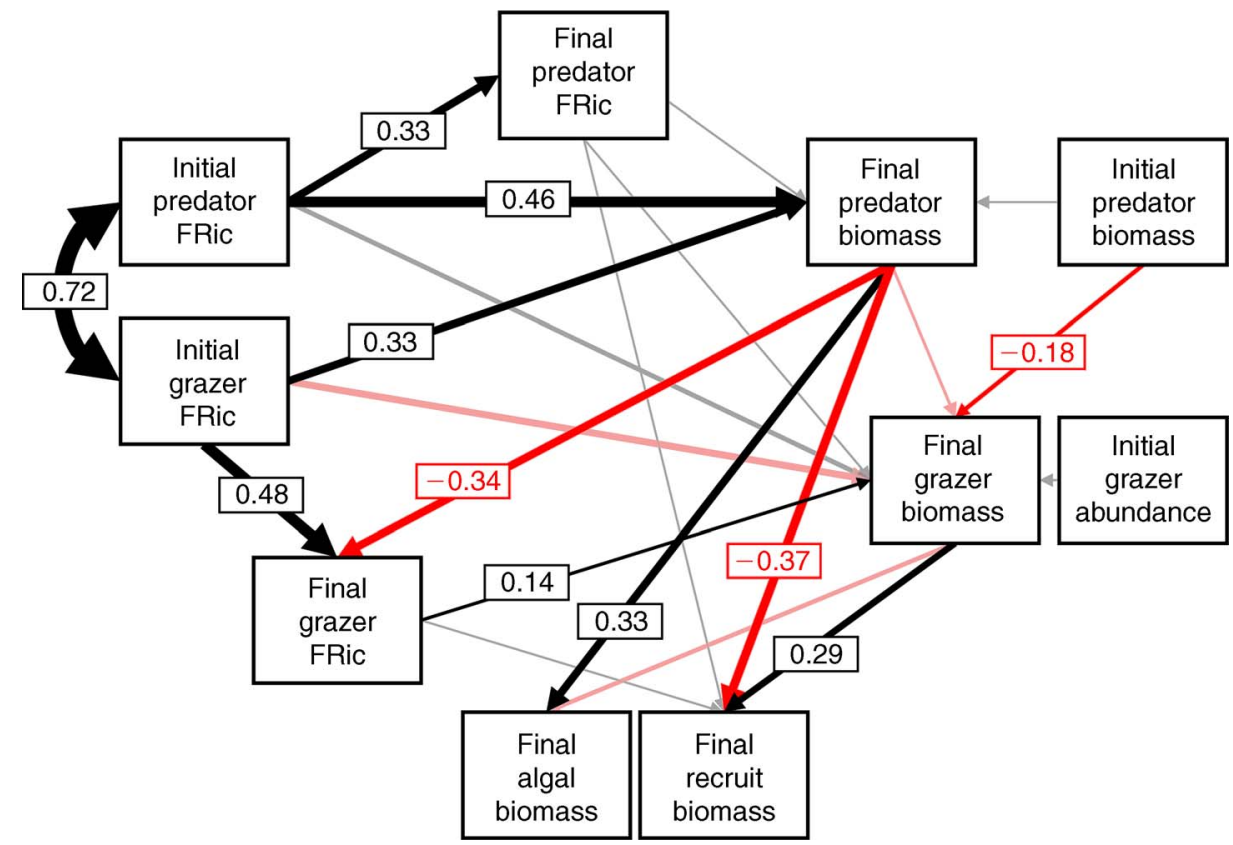

FIG. 3. Structural equation model of herbivore and predator functional diversity (functional richness, FRic) as a predictor of community responses at the end of the experiment. Black arrows represent positive paths, and red arrows represent negative paths. Arrow width is proportional to the size of the effect, reported as the standardized effect size in the accompanying text box. Lightly shaded lines represent nonsignificant paths $(P \geq 0.05$, Appendix A: Table A4). 


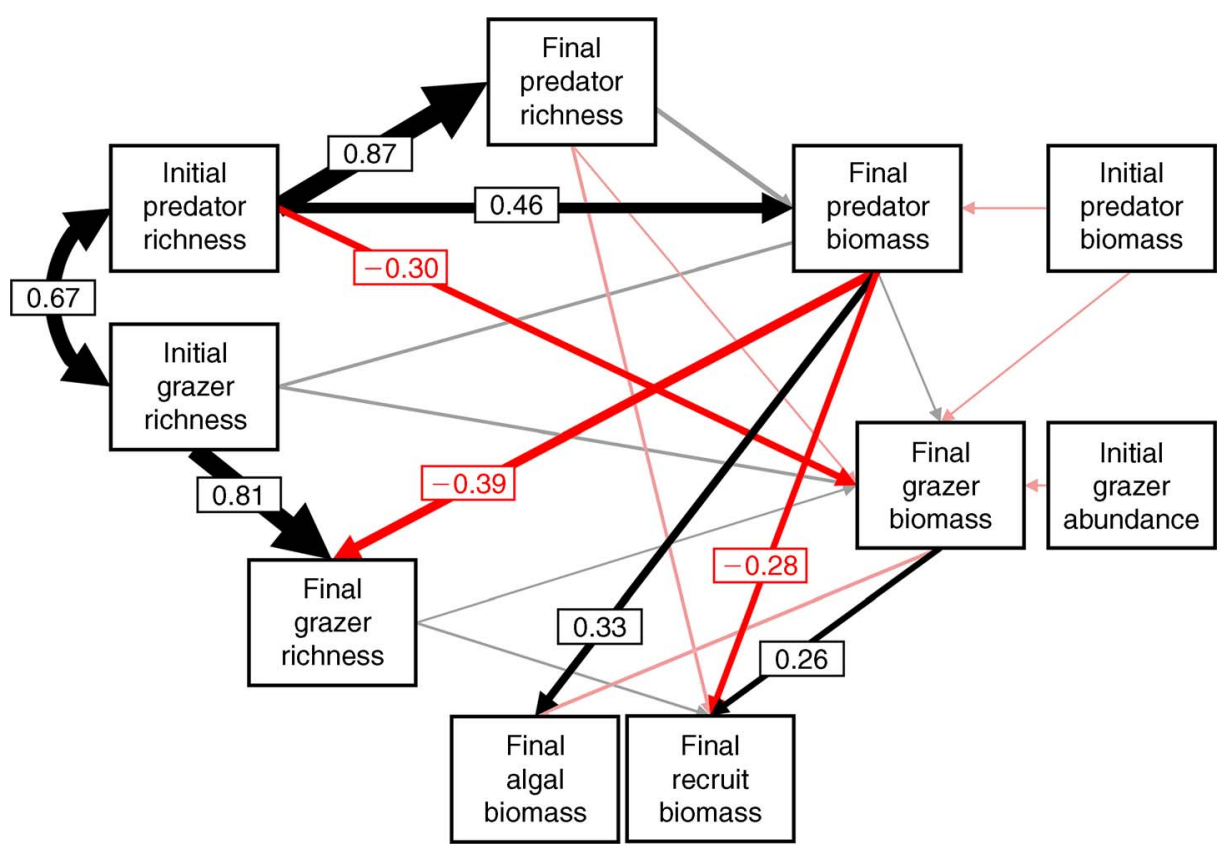

FIG. 4. Structural equation model of herbivore and predator species richness as a predictor of community responses at the end of the experiment. Black arrows represent positive paths, and red arrows represent negative paths. Arrow width is proportional to the size of the effect, reported as the standardized effect size in the accompanying text box. Lightly shaded lines represent nonsignificant paths $(P \geq 0.05$, Appendix A: Table A5).

FD and final grazer biomass, accounting for the other covariates in the SEM (Fig. A5). This relationship was still significant and more than double in magnitude when considering replicates that only contained grazers ( $\beta=0.353, P=0.048)$.

Most interestingly, there was no direct effect of final predator biomass on final grazer biomass, but rather this relationship was mediated via a reduction in final grazer FD (Fig. 3). The magnitude of this indirect effect is achieved by multiplying the two component paths: $\beta=$ $-0.335 \times 0.142=-0.048$, indicating a weak but still significant decrease. Additionally, initial predator biomass weakly reduced final grazer biomass $(\beta=-0.180, P$ $=0.028$, Fig. 3), suggesting that grazer communities experienced rapid top-down control by predators, and only after prey communities had stabilized that grazer FD increased grazer biomass.

In contrast, the SEM using species richness as the metric of diversity was a much poorer fit to the data than the SEM using FD $\left(C_{36}=39.49, P=0.317\right.$, Fig. 4). Comparison of AIC scores revealed that the SEM based on FD was also much likelier than the one based on species richness (AIC $=106.7 \ll 121.5$ for FD vs. richness). The most striking difference between the two models was the lack of a significant effect of either initial grazer richness $(\beta=0.145, P=0.335)$ or final grazer richness on grazer biomass $(\beta=0.059, P=$ 0.457 , Fig. 4). Additionally, there was no significant relationship between initial grazer richness and final predator biomass $(\beta=0.154, P=0.115$, Fig. 4$)$, whereas there was a positive and significant bottom-up path from initial grazer functional diversity to final predator biomass in the FD SEM $(\beta=0.327, P=$ 0.007 , Fig. 3). Finally, in the richness SEM, the primary top-down path manifested directly between initial predator richness and final grazer biomass $(\beta=$ $-0.303, P=0.037$, Fig. 4), although it was approximately equal in magnitude than the corresponding effect of final predator biomass on final grazer functional diversity in the FD SEM $(\beta=-0.335, P=$ 0.017, Fig. 3). All coefficients and their associated $P$ values for both the FD and richness SEMs are given in Appendix A: Tables A4 and A5.

The individual contributions of each species to functioning revealed potential for complementarity across multiple functions (Table 2). As expected, most of the grazers positively and significantly contributed to final grazer biomass, with the exception of E. attenuata. Similarly, the two fishes F. heteroclitus and Syngnathus spp. both contributed positively to final predator biomass. The mummichog $F$. heteroclitus also significantly reduced recruiting invertebrate biomass, and Ampithoid spp. contributed significantly to reductions in final algal and Gracilaria biomass. Regression of the effect sizes in Table 2 against functional distinctnesscalculated as the mean pairwise distance between a given species and all other species in multidimensional trait space - revealed that some functions were driven largebodied, mobile predators, while others were driven by small-bodied, chitinous grazers (Fig. A6). 
TABLE 2. Standardized contributions of individual species to ecosystem responses based on regressions of presence/absence of each species against a given response.

\begin{tabular}{|c|c|c|c|c|c|c|c|c|c|}
\hline Response & Amp & Bitt & Call & Erich & Fund & Gamm & Hippo & Pal & Syn \\
\hline Final grazer biomass & 1.33 & -0.57 & -0.29 & 0.10 & -0.68 & 0.69 & -0.53 & -0.34 & 0.22 \\
\hline Final predator biomass & 0.12 & 0.17 & 0.30 & 0.15 & 1.19 & 0.06 & -0.03 & 0.15 & 0.45 \\
\hline Recruit invert biomass & 0.41 & -0.45 & -0.24 & 0.24 & -0.70 & -0.03 & -0.35 & -0.09 & -0.06 \\
\hline Final algal biomass & -0.60 & 0.07 & -0.30 & -0.15 & 0.01 & 0.17 & 0.59 & 0.03 & 0.01 \\
\hline Final Gracilaria biomass & -0.73 & 0.09 & 0.54 & 0.30 & 0.33 & 0.00 & 0.18 & 0.37 & -0.28 \\
\hline
\end{tabular}

Notes: Significant effects $(P<0.05)$ are shown in boldface type. Species abbreviations are Amp, Ampithoid spp.; Bitt, Bittiolum varium; Call, Callinectes sapidus; Erich, Erichsonella attenuate; Fund, Fundulus heteroclitus; Gamm, Gammarus mucronatus; Hippo, Hippolyte pleuracanthus; Pal, Palaemonetes pugio; and Syn, Syngnathus spp.

\section{DisCUSSION}

In this study of an estuarine food web, we found that multivariate functional diversity better predicted standing stock biomass across multiple trophic levels than did species richness (Table 1). This result was a consequence of greater variation in FD than in richness (Appendix A: Fig. A3), confirming the superior utility of FD for capturing ecologically significant variation among members of an assemblage compared to the raw number of species. Further, structural equation modeling (SEM) revealed that the FD but not richness of grazers and especially predators enhanced corresponding biomass, emphasizing the important influence of multitrophic diversity on community structure and functioning (Strong 1992, Polis and Strong 1996). Finally, and notably, the negative effect of predators on grazer biomass in our experiment was not direct, but rather mediated through a reduction in grazer FD, which translated to lower biomass (Fig. 3).

Contrary to our predictions, we did not find a significant interaction between species richness and FD for most ecosystem responses, though initial species richness and functional diversity had antagonistic effects on final species richness (Table A2). This may have been due to the high collinearity between species richness and functional diversity inflating standard errors of our model predictions $(r=0.94$, Fig. A3). Despite this potential conservative bias, we were still able to isolate a significant main effect of FD, but not species richness. Thus, in our experiment, the effect of increasing FD on grazer, predator, and recruiting invertebrate biomass appeared not to be contingent on the level of species richness. One explanation may be our experimental design, which nested two levels of FD within only two levels of species richness (Fig. A1). There may have been too few levels of species richness, or too little variation among species' functional traits, to extract a clearer signal. Future manipulations may benefit from incorporating an even greater range of species richness and/or traits in investigation of the diversity-function relationship (Gamfeldt et al. 2015).

The stronger effects of predator FD compared relative to herbivores (Fig. 3) is consistent with both conceptual predictions relating to greater physiological, resource, and behavioral complexity with increasing trophic level (Duffy 2002), as well as experimental evidence (Griffin et al. 2013, Gamfeldt et al. 2015, Lefcheck et al. 2015). A possible explanation for the strong predator diversity effect in our experiment is that the predator species was more functionally distinct, on average, than the grazer species (functional distinctness for predators $=0.55 \pm$ 0.06 vs. $0.45 \pm 0.02$ for grazers [mean $\pm \mathrm{SE}]$ ), enhancing the potential for resource complementarity among predators (e.g., Griffin et al. 2008). This distinctness, however, appears to be driven largely by $F$. heteroclitus (Fig. A6), which also happens to have the largest significant effects of all the predators on the ecosystem responses (Table 2). Thus, the stronger effect of predator diversity relative to herbivore diversity may best be interpreted as a sampling effect (sensu Loreau 1998), driven by the presence of $F$. heteroclitus. This result speaks to the central role of $F$. heteroclitus in the food web dynamics of estuarine systems in the southeastern United States (Kneib 1986).

In addition to positive effects of FD on biomass within trophic levels and the top-down effect of predators on grazer biomass through grazer FD, we observed that initial grazer FD, but not initial species richness, increased final predator biomass (Fig. A7). These results confirm as expected that it is not just the total number of species, but also the functional identity of the grazers that are important in mediating predatorprey interactions. Our exploration of individual traits revealed that the predictive ability of multivariate FD decreased significantly when body plan and trophic level were left out of the index (Table A3), implying that variation in these traits was central in determining final predator biomass. It is not surprising that these traits come out as being particularly important, as they are central to classical habitat-based (Grinnell 1917) and resource-based definitions of ecological niches (Elton 1927). One possible mechanistic explanation is that body plan influenced susceptibility to predation. For instance, the long body of the isopod E. attenuata may exceed the gape limit of the pipefish Syngnathus spp., but could more easily be manipulated by the crab C. sapidus. Thus, differences in morphology may drive predatorspecific selection of prey, and ultimately increase aggregate consumption across a variety of prey body types in diverse assemblages.

Variation in trophic level may have been important in determining final predator biomass simply because high 
variation implies the presence more than one trophic level, i.e., predators and their prey. A related explanation for the positive effect of grazer functional diversity on predator biomass could be the "balanced diet" hypothesis, where a diverse prey assemblage provides a more complete range of nutrients (Gamfeldt et al. 2005, Lefcheck et al. 2013). If prey species varied slightly in their positions within the food web, then they may be assimilating resources differently. For instance, the ampithoid amphipod complex, principally C. compta, was the only grazer to have a detectable negative effect on primary producers in our experiment by directly consuming algal species (Table 2), and previous experiments also documented distinct differences in diet between Ampithoid spp. and another amphipod grazer used in our experiment, G. mucronatus (Duffy and Harvilicz 2001). The positive effect of grazer functional diversity may thus indicate niche complementarity increasing aggregate biomass and/or nutritional value (Fig. 3, Fig. A5), leading to more, and potentially more nutritious, prey for predators.

That the top-down effects of predators on grazers did not cascade to primary producers or recruiting invertebrate biomass was surprising, given both theoretical predictions (Strong 1992) and past experiments with these grazers (Duffy et al. 2003, 2005, O'Connor and Bruno 2007, Douglass et al. 2008). One possible explanation is that predators were simply so efficient that our model was unable to disentangle the indirect effects of predators removing grazers and subsequent release of primary producers, leading to the strong direct positive path between predator biomass and final algal biomass in our SEMs (Figs. 3, 4). Similarly, while grazers have been shown to influence the recruiting invertebrates in mesocosms in the absence of predators (e.g., Duffy et al. 2003), their effect relative to larger predators was insignificant in our experiment (Table 2). This was almost certainly due to the presence of known generalists such as $C$. sapidus and $F$. heteroclitus, and possibly also a consequence of the rapid consumption of grazers (Fig. 1a), limiting their potential to interact with recruiting invertebrates. Thus, there was also a direct negative relationship between predator biomass and recruiting invertebrate biomass (Figs. 3, 4).

Our exploration of individual traits revealed that no single trait was responsible for driving the patterns in multivariate FD. Rather, all traits showed generally similar trends to multivariate FD in influencing final biomass (Fig. 2). While this analysis confirmed that there were not strong trade-offs among individual traits that may have biased the multivariate trend, it also raises the question: why combine inferences from multiple traits at all? One answer is that the multivariate index generally had lower predicted standard errors (Fig. A4), thus improving prediction accuracy. In some cases, the multivariate index reduced standard errors on estimates of grazer and recruiting invertebrate biomass by up to $40 \%$, particularly when considering only armor, body plan, and trophic level. This result also explains why models dropping these two traits generally had worse AIC scores when attempting to predict final predator biomass (Table A3). Thus, combining multiple traits enhanced the explanatory power of functional diversity, at least in our index of functional richness.

Richness and FD of species stocked in our mesocosms were lower at the end of the experiment than at the beginning (Fig. A8), highlighting the negative interactions among predators and grazers, and potentially among predators. For instance, blue crabs were lost in several replicates, leading to the overall nonsignificant effect of blue crabs on every ecosystem response (Table 2 ). The loss of $C$. sapidus corresponds with other experiments using this species (O'Connor and Bruno 2007, Douglass et al. 2008), and was partly due to crabs escaping the experimental mesocosms, and partly due to the death of crabs, as evidenced by empty carapaces found in the mesocosms at the end of the experiment. While there could have been antagonistic interactions among predators, all crabs were recovered from the polycultures, and virtually none from the monocultures. Cannibalism is not a likely explanation as crabs, like all predators, were stocked individually. This result contrasts that of Douglass et al. (2008), who found that crab growth and survival was highest in monoculture. They attributed this result to the presence of other predators modifying grazer composition to the detriment of blue crabs. The nonrandom pattern of crab loss across the treatments in this study suggests the opposite: that only the diverse assemblage provided the requisite resources for blue crab survival. This idea is bolstered by the finding that the nine-species mixture retained a higher number of stocked species in general (Fig. A8a). Functional richness also enhanced final diversity, as measured by both final species richness and final function richness (Fig. A8). Thus, our results also suggest that more functionally diverse communities were also more stable, retaining a larger proportion of stocked species over the course of the experiment.

Overall, this study empirically confirms that a focus on multiple functional traits can provide more accurate predictions regarding the functioning of whole food webs than single traits or species richness alone. Moreover, we show that functional diversity within multiple trophic levels (herbivores and predators) enhanced corresponding biomass even after accounting for the effects of adjacent trophic levels. This result suggests that conservation of diversity at multiple trophic levels, with a particular emphasis functional variation among species, can lead to enhanced community biomass.

\section{ACKNOWLEDGMENTS}

We thank K. M. Laumann, S. Donadi, J. P. Richardson, K. Jenkins, and Berlioz for support during the experiment. We additionally thank J. N. Griffin, G. E. Edgar, J. J. Orth, and two anonymous reviewers for comments on previous versions of the manuscript. J. S. Lefcheck was supported by VIMS 
Council and Maury Fellowships. J. E. Duffy and J. S. Lefcheck were supported by NSF OCE-1031061 to J. E. Duffy. This is Contribution No. 3465 of the Virginia Institute of Marine Science, College of William and Mary.

\section{Literature Cited}

Best, R. J., N. C. Caulk, and J. J. Stachowicz. 2013. Trait vs. phylogenetic diversity as predictors of competition and community composition in herbivorous marine amphipods. Ecology Letters 16:72-80.

Borer, E. T., B. S. Halpern, and E. W. Seabloom. 2006. Asymmetry in community regulation: effects of predators and productivity. Ecology 87:2813-2820.

Bremner, J., S. I. Rogers, and C. L. J. Frid. 2003. Assessing functional diversity in marine benthic ecosystems: a comparison of approaches. Marine Ecology Progress Series 254:1125.

Bruno, J. F., K. E. Boyer, J. E. Duffy, and S. C. Lee. 2008. Relative and interactive effects of plant and grazer richness in a benthic marine community. Ecology 89:2518-2528.

Bruno, J. F., and M. I. O'Connor. 2005. Cascading effects of predator diversity and omnivory in a marine food web. Ecology Letters 8:1048-1056.

Burnham, K. P., and D. R. Anderson. 2002. Model selection and multimodel inference. Second edition. Springer-Verlag, New York, New York, USA.

Cardinale, B. J., et al. 2012. Biodiversity loss and its impact on humanity. Nature 486:59-67.

Cardinale, B. J., D. S. Srivastava, J. E. Duffy, J. P. Wright, A. L. Downing, M. Sankaran, and C. Jouseau. 2006. Effects of biodiversity on the functioning of trophic groups and ecosystems. Nature 443:989-992.

Díaz, S., and M. Cabido. 2001. Vive la difference: plant functional diversity matters to ecosystem processes. Trends in Ecology \& Evolution 16:646-655.

Douglass, J. G., J. E. Duffy, and J. F. Bruno. 2008. Herbivore and predator diversity interactively affect ecosystem properties in an experimental marine community. Ecology Letters 11:598-608.

Douglass, J. G., J. Emmett Duffy, and E. A. Canuel. 2011. Food web structure in a Chesapeake Bay eelgrass bed as determined through gut contents and ${ }^{13} \mathrm{C}$ and ${ }^{15} \mathrm{~N}$ isotope analysis. Estuaries and Coasts 34:701-711.

Douglass, J. G., K. E. France, J. Paul Richardson, and J. E. Duffy. 2010. Seasonal and interannual changes in a Chesapeake Bay eelgrass community: insights into biotic and abiotic control of community structure. Limnology and Oceanography 55:1499-1520.

Duffy, J. E. 2002. Biodiversity and ecosystem function: the consumer connection. Oikos 99:201-219.

Duffy, J. E., B. J. Cardinale, K. E. France, P. B. McIntyre, E. Thébault, and M. Loreau. 2007. The functional role of biodiversity in ecosystems: incorporating trophic complexity. Ecology Letters 10:522-538.

Duffy, J. E., and A. M. Harvilicz. 2001. Species-specific impacts of grazing amphipods in an eelgrass-bed community. Marine Ecology Progress Series 223:201-211.

Duffy, J. E., J. P. Richardson, and E. A. Canuel. 2003. Grazer diversity effects on ecosystem functioning in seagrass beds. Ecology Letters 6:637-645.

Duffy, J. E., J. P. Richardson, and K. E. France. 2005. Ecosystem consequences of diversity depend on food chain length in estuarine vegetation. Ecology Letters 8:301-309.

Edgar, G. J. 1990. The use of the size structure of benthic macrofaunal communities to estimate faunal biomass and secondary production. Journal of Experimental Marine Biology and Ecology 137:195-214.

Elton, C. 1927. Animal ecology. Macmillan, New York, New York, USA.

Finke, D. L., and R. F. Denno. 2004. Predator diversity dampens trophic cascades. Nature 429:22-24.
Flynn, D. F. B., N. Mirotchnick, M. Jain, M. I. Palmer, and S. Naeem. 2011. Functional and phylogenetic diversity as predictors of biodiversity-ecosystem-function relationships. Ecology 92:1573-1581.

Fox, J. W. 2004. Modelling the joint effects of predator and prey diversity on total prey biomass. Journal of Animal Ecology 73:88-96.

Gagic, V., et al. 2015. Functional identity and diversity of animals predict ecosystem functioning better than speciesbased indices. Proceedings of the Royal Society B 282:2014.2620.

Gamfeldt, L., H. Hillebrand, and P. R. Jonsson. 2005. Species richness changes across two trophic levels simultaneously affect prey and consumer biomass. Ecology Letters 8:696703.

Gamfeldt, L., J. S. Lefcheck, J. E. K. Byrnes, B. J. Cardinale, J. E. Duffy, and J. N. Griffin. 2015. Marine biodiversity and ecosystem functioning: what's known and what's next. Oikos 124:252-265.

Grace, J. B. 2006. Structural equation modeling and natural systems. Cambridge University Press, New York, New York, USA.

Grace, J. B., D. R. Schoolmaster, Jr., G. R. Guntenspergen, A. M. Little, B. R. Mitchell, K. M. Miller, and E. W. Schweiger. 2012. Guidelines for a graph-theoretic implementation of structural equation modeling. Ecosphere 3:1-44.

Griffin, J. N., J. E. K. Byrnes, and B. J. Cardinale. 2013. Effects of predator richness on prey suppression: a meta-analysis. Ecology 94:2180-2187.

Griffin, J. N., K. L. de la Haye, S. J. Hawkins, R. C. Thompson, and S. R. Jenkins. 2008. Predator diversity and ecosystem functioning: density modifies the effect of resource partitioning. Ecology 89:298-305.

Griffin, J. N., V. Méndez, A. F. Johnson, S. R. Jenkins, and A. Foggo. 2009. Functional diversity predicts overyielding effect of species combination on primary productivity. Oikos 118: $37-44$.

Grinnell, J. 1917. The niche-relationships of the California Thrasher. Auk 34:427-433.

Hooper, D. U., et al. 2005. Effects of biodiversity on ecosystem functioning: a consensus of current knowledge. Ecological Monographs 75:3-35.

Isbell, F., et al. 2011. High plant diversity is needed to maintain ecosystem services. Nature 477:199-202.

Kneib, R. T. 1986. The role of Fundulus heteroclitus in salt marsh trophic dynamics. American Zoologist 269:259-269.

Laliberté, E., P. Legendre, and B. Shipley. 2014. FD: measuring functional diversity from multiple traits, and other tools for functional ecology. R package version 1.0-12. http://cran. r-project.org/web/packages/FD/index.html

Lefcheck, J. S. 2015. Top-down versus bottom-up control of a temperate eelgrass bed: insights from a 15-year ecological survey. Dissertation. Virginia Institute of Marine Science, The College of William and Mary, Gloucester Point, Virginia, USA.

Lefcheck, J. S., J. E. K. Byrnes, F. Isbell, L. Gamfeldt, J. N. Griffin, N. Eisenhauer, M. J. S. Hensel, A. Hector, B. J. Cardinale, and J. E. Duffy. 2015. Biodiversity enhances ecosystem multifunctionality across trophic levels and habitats. Nature Communications 6:6936.

Lefcheck, J. S., M. A. Whalen, T. M. Davenport, J. P. Stone, and J. E. Duffy. 2013. Physiological effects of diet mixing on consumer fitness: a meta-analysis. Ecology 94:565-572.

Loreau, M. 1998. Separating sampling and other effects in biodiversity experiments. Oikos 82:600-603.

Mouillot, D., M. Scherer-Lorenzen, S. Villéger, and N. W. H. Mason. 2011. Functional structure of biological communities predicts ecosystem multifunctionality. PLoS ONE 6:e17476.

Nakagawa, S., and H. Schielzeth. 2012. A general and simple method for obtaining $R^{2}$ from generalized linear mixed- 
effects models. Methods in Ecology and Evolution 4:133142.

O'Connor, M. I., and J. F. Bruno. 2009. Predator richness has no effect in a diverse marine food web. Journal of Animal Ecology 78:732-740.

O'Connor, N. E., and J. F. Bruno. 2007. Predatory fish loss affects the structure and functioning of a model marine food web. Oikos 116:2027-2038.

Parker, J. D., J. E. Duffy, and R. J. Orth. 2001. Plant species diversity and composition: experimental effects on marine epifaunal assemblages. Marine Ecology Progress Series 224: $55-67$.

Petchey, O. L., and K. J. Gaston. 2002. Functional diversity (FD), species richness and community composition. Ecology Letters 5:402-411.

Petchey, O. L., A. Hector, and K. J. Gaston. 2004. How do different measures of functional diversity perform? Ecology 85:847-857.

Pinheiro, J., D. Bates, S. DebRoy, D. Sarkar, and R Core Team. 2013. nlme: linear and nonlinear mixed effects models. http://CRAN.R-project.org/package=nlme

Podani, J. 1999. Extending Gower's general coefficient of similarity to ordinal characters. Taxon 48:331-340.

Polis, G. A., and D. R. Strong. 1996. Food web complexity and community dynamics. American Naturalist 147:813.

Reiss, J., J. R. Bridle, J. M. Montoya, and G. Woodward. 2009. Emerging horizons in biodiversity and ecosystem functioning research. Trends in Ecology \& Evolution 24:505-514.

Rosenfeld, J. S. 2002. Functional redundancy in ecology and conservation. Oikos 98:156-162.

Schittko, C., M. Hawa, and S. Wurst. 2014. Using a multi-trait approach to manipulate plant functional diversity in a biodiversity-ecosystem function experiment. PLoS ONE 9: e99065.

Schmitz, O. J. 2008. Effects of predator hunting mode on grassland ecosystem function. Science 319:952-954.

Shipley, B. 2009. Confirmatory path analysis in a generalized multilevel context. Ecology 90:363-368.

Shipley, B. 2013. The AIC model selection method applied to path analytic models compared using a d-separation test. Ecology 94:560-564.

Shurin, J. B., S. Mandal, and R. L. Abbott. 2014. Trait diversity enhances yield in algal biofuel assemblages. Journal of Applied Ecology 51:603-611.

Spasojevic, M. J., and K. N. Suding. 2012. Inferring community assembly mechanisms from functional diversity patterns: the importance of multiple assembly processes. Journal of Ecology 100:652-661.

Straub, C. S., and W. E. Snyder. 2006. Species identity dominates the relationship between predator biodiversity and herbivore suppression. Ecology 87:277-282.

Strong, D. R. 1992. Are trophic cascades all wet? Differentiation and donor-control in speciose ecosystems. Ecology 73: $747-754$.

van Montfrans, J., R. L. Wetzel, and R. J. Orth. 1984. Epiphyte-grazer relationships in seagrass meadows: consequences for seagrass growth and production. Estuaries 7: 289-309.

Villéger, S., N. W. H. Mason, and D. Mouillot. 2008. New multidimensional functional diversity indices for a multifaceted framework in functional ecology. Ecology 89:22902301 .

\section{Supplemental Material}

\section{Ecological Archives}

Appendices A and B and the Supplement are available online: http://dx.doi.org/10.1890/14-1977.1.sm 